\title{
[Preprint]
}

\section{Review on the Methods for Evaluation of Root Reinforcement in Shallow Landslides}

\author{
Ana Sofia Dias ${ }^{1,2}$, Marianna Pirone $^{1}$, and Gianfranco Urciuoli ${ }^{1}$ \\ ${ }^{1}$ Department of Civil, Building and Environmental Engineering, University of Naples Federico II, Via \\ Claudio 21, 80125 Naples, Italy \\ ${ }^{2}$ AMAP, INRA, IRD, CNRS, CIRAD, University of Montpellier, Montpellier, France
}

May 29, 2017

Conference: Workshop on World Landslide Forum

Title of the book: Advancing Culture of Living with Landslides

\begin{abstract}
Recently geotechnical engineers aim to adopt more environmental-friendly solutions (not harmful to the environment), therefore the interest on the use of vegetation as a measure to improve slope stability is increasing. The mechanical reinforcement due to roots against shallow landslides occurs when the fibres intersect the shear surface, usually at depths lower than $2 \mathrm{~m}$. In the literature, the presence of roots is often taken into account by modelling the soil as an equivalent composite material: 'the root-permeated soil', by including an additional cohesion term in the Mohr-Coulomb equation. The models used to estimate the root additional cohesion are presented in the first part of the paper. In some cases, root cohesion is calculated based on the resistant properties of the fibres and assuming an order for the progressive roots failure, either breaking, slipping out or buckling. On the other hand, some authors used structural models of the roots investigating not only the stresses in the roots, but also in the surrounding soil to obtain a better estimation of the root cohesion. In the second part of the paper, the calculation of the root reinforcement is used to assess the safety factor (SF) of the slope. Both Limit Equilibrium analyses (LE) and Finite Element Methods (FEM) are discussed, stressing the limitations of both the approaches.
\end{abstract}

\section{Keywords}

Root cohesion, Root mechanical reinforcement, Shallow landslides, Slope stability 


\section{Introduction}

The shallow landslide's failure surface reaches depths of up to $2 \mathrm{~m}$ and it can intersect roots, which provide an increase of soil resistance. Therefore, the introduction of vegetation in a slope is an appealing measure to increase the slope safety.

The way roots influence the behaviour of the composite material (root-permeated soil) was investigated by Graf et al. (2009) and Zhang et al. (2010), however, the root reinforcement is usually modelled as an additional cohesion term (Wu 1976). Nevertheless, the type of root failure cannot be neglected because the ultimate load taken by a root depends on the rupture mechanism (Wu et al. 1988; Schwarz et al. 2015).

Soil-root interaction models for the quantification of such reinforcement are presented in this paper. One approach can be the estimation of the maximum reinforcement by bundle of roots as a sum of the resistance provided each individual (Wu 1976; Pollen and Simon 2005; Schwarz et al. 2010a, 2013). Some other authors used numerical models, which consider the root and soil influence on each other (Mao et al. 2014; Liang et al. 2015).

Moreover, the root reinforcement for stability of shallow landslides can be evaluated by either a macro model or a soil-root interaction model (Wu 2013; Cazzuffi et al. 2014). The first is preferable in the case of roots with small dimensions and spacing. The rooted soil is represented by a homogeneous material with given resistant properties obtained from tests on root-permeated soil. The second is more convenient when roots are large and disperse, so that they are investigated as soil-embedded elements allowing the estimation of the stresses in the fibre.

The objective of the paper is to present the approaches adopted in the literature to analyse root reinforcement and its use on assessing slope stability. The advantages and disadvantages of the methods, as well as theirs limitations, concerns and future work projections are discussed.

\section{Roots Effect on Soil Shear Resistance}

The root-permeated soil is a composite material whose shear strength is described by (1) (Veylon et al. 2015). This way, the shear stress at failure $\left(s_{f}\right)$ is a sum of the effective shear strength of the soil matrix given by Mohr-Coulomb criterion $\left(s_{s}\right)$, shear strength component due to matric suction $\left(s_{\psi}\right)$ and shear strength component due to the root system $\left(s_{r}\right)$.

$$
s_{f}=s_{s}+s_{\psi}+s_{r}
$$

In the majority of the literature, roots are considered to provide increase of cohesion to the soil. The works stating that roots influence the composite material's friction angle are very scarce. Triaxial tests were performed by Graf et al. (2009) and Zhang et al. (2010) reporting opposite conclusions regarding the roots effect on the soil resistance.

Graf et al. (2009) performed consolidated-undrained (CU) triaxial tests on vegetated soil (clayey gravel with sand) in saturated conditions applying confining pressures of 50,75 and $100 \mathrm{kPa}$ to represent the low stresses of surface soil. The results showed that roots provide an increase of internal friction angle of up to $5^{\circ}$ (from $34.3^{\circ}$ to $39.4^{\circ}$ ) but no additional cohesion. Therefore, Graf et al. (2009) showed dissatisfaction about considering the effect of roots as an additional cohesion, as typically is assumed, as the effect of roots should rather be a stress-dependent term.

In the results of direct shear tests of Fig. 1, the parallel displacement of shear envelopes suggested that fibres do not affect the frictional properties of the soil (sand). Yet, it is interesting to notice that 
for the range of normal stresses used by Graf et al. (2009), Fig. 1 shows an increase of the internal friction angle (Gray and Ohashi 1983).

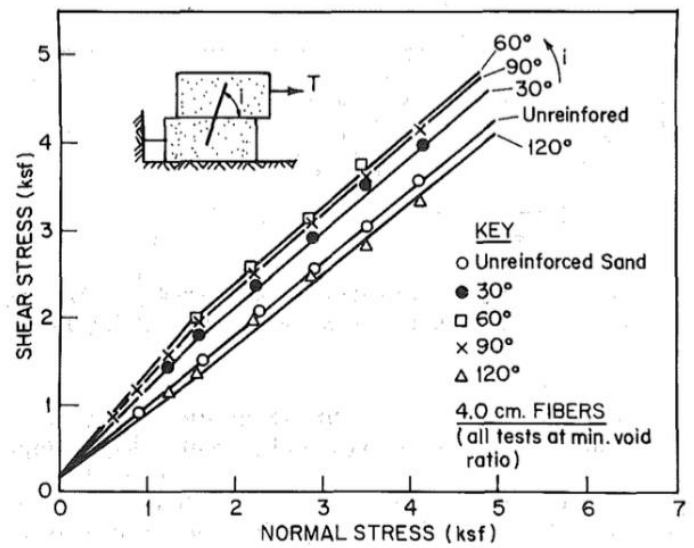

Fig. 1 Influence of fibres with different orientations on the shear strength envelope $\left(1 \mathrm{ksf}=47.9 \mathrm{kN} / \mathrm{m}^{2}\right)($ Gray and Ohashi 1983)

On the other hand, the consolidated-drained (CD) triaxial tests performed by Zhang et al. (2010) on rooted soil (sand) with different water contents and roots in different directions showed that roots increased soil shear strength by increasing cohesion.

In the works of Graf et al. (2009) and Zhang et al. (2010), macro models of root-permeated soil were obtained according with the classification of $\mathrm{Wu}$ (2013) because the stresses in the roots were not investigated.

\section{Root Cohesion}

As generally accepted, the root shear resistance $s_{r}$ of (1) is an additional cohesion. However, roots are elements with low bending and shear resistance, and Wu (1976) proposed the following soil-root interaction model (Fig. 2). The shearing on the sliding surface causes the elongation of the roots, which are subjected to tensile stress and act on the unstable volume as stabilizing external forces. The normal component of the root tensile stress $(\mathrm{Tn})$ increases the confining stress on the failure plane, thereby mobilizing additional shear resistance in the soil, whereas the tangential component ( $\mathrm{Tt})$ directly resists shear. The increase of shear strength provided by the root $s_{r}$ may be translated by (2), where $\theta$ is the shear distortion of the root (Fig. 2) and $t_{r}$ is the root tensile resistance per unit area of soil.

$$
s_{r}=\left(\sin \theta+\cos \theta \tan \phi^{\prime}\right) t_{r}=k^{\prime} t_{r}
$$

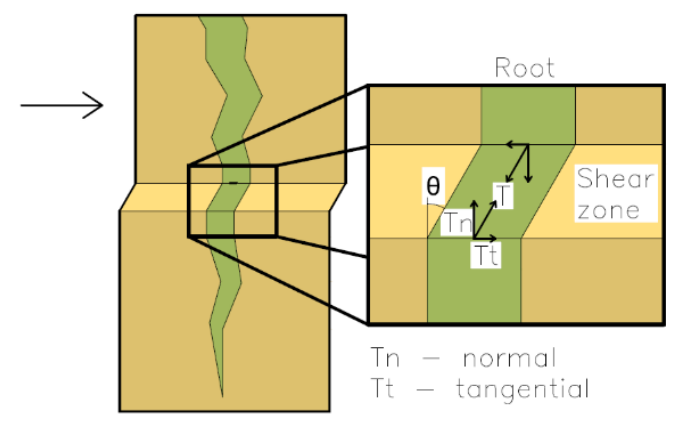

Fig. 2 Scheme of the stresses in the root during shear 
The value of the angle $\theta$ is difficult to quantify, so $\mathrm{Wu}(1976)$ suggested the use of a coefficient $k^{\prime}$ that takes the value of 1.2. Indeed, the approximation proposed by $\mathrm{Wu}$ (1976) is generally accepted because it was observed in laboratory and field investigations that $k^{\prime}$ ranges between 1.0 and 1.3. However, Thomas and Pollen-Bankhead (2010) recommended the use of a $k^{\prime}$ close to 1.0 since that the parameter $k^{\prime}$ also intends to take into account the initial angle between the root and the shear surface because the root is tensioned when it is inclined towards the movement direction. When the roots are inclined opposing the movement of the upper layer, the root is subjected to compression providing less resistance.

Wu et al. (1988), Abe and Ziemer (1991) and Belfiore and Urciuoli (2004) also made some considerations on the simplification proposed by $\mathrm{Wu}(1976)$ because the angle $\theta$ is the parameter that directly controls the tension in the roots, on which depends the root elongation. Improvements have been done to this formulation mainly to take into account the soil-root surface interaction, root inclination and deformation.

In order to compute the root reinforcement in the form of root additional cohesion, the model presented in Fig. 2 needs the tensile resistance of the roots present in the bundle, i.e. $t_{r}$, as an input. Several authors proposed models to quantify this parameter. The main difference among them is the order by which roots fail, which leads to different values of root reinforcement. This models are referred as root "breaking" models (BM) in this paper. The term "breaking" regards to the failure of the root, either by breakage, slippage or buckling.

Some other authors used numerical models to obtain the reinforcement of the roots also in terms of additional cohesion (Mao et al. 2014; Liang et al. 2015). In the following both "breaking" models and numerical ones will be discussed.

\section{Root "Breaking" Models}

\section{W\&W Model}

The model commonly called W\&W was first formulated by Wu (1976). It is assumed that all the roots break simultaneously and pull out does not occur. Therefore, the tensile strength of root fibres per unit area of soil $t_{r}$ is given by (3), representing the sum of the maximum tensile strength of each individual root. For simplification, the roots are grouped in $N$ diameter classes so that the value of $t_{r, n}$ is the average tensile strength of the root class, $R A R_{n}$ is the root area ratio of the entire class $n$ (the root area ratio is the fraction of a given control area that is occupied by roots).

$$
t_{r}=\sum_{n=1}^{N} t_{r, n} \cdot R A R_{n}
$$

This model tends to overestimate the root reinforcement because roots do not break simultaneously (Pollen and Simon 2005; Thomas and Pollen-Bankhead 2010). Better estimations are obtained for grass root systems because the root diameters are more homogeneous (Thomas and Pollen-Bankhead 2010). The value obtained from (3) is substituted into (2) to compute the root reinforcement.

\section{Fibre Bundle Model}

In order to avoid some limitations of the W\&W model, Pollen and Simon (2005) introduced the Fibre Bundle Model (FBM). The maximum load withstood by the bundle of fibres is less than the sum of each of their individual strengths because roots break at different instants depending on the way the load is distributed by all the fibres. Therefore the maximum value of tensile strength provided by the bundle not to be the same as if all the roots provided their maximum strength at the same instant. 
It is assumed in the FBM that all fibres have the same elastic properties, the complexity of root tortuosity is not considered and all roots break rather than pull out of the soil. The computation of the breakage sequence in the FBM follows the flowchart presented in Fig. 3 (Mao et al. 2012).

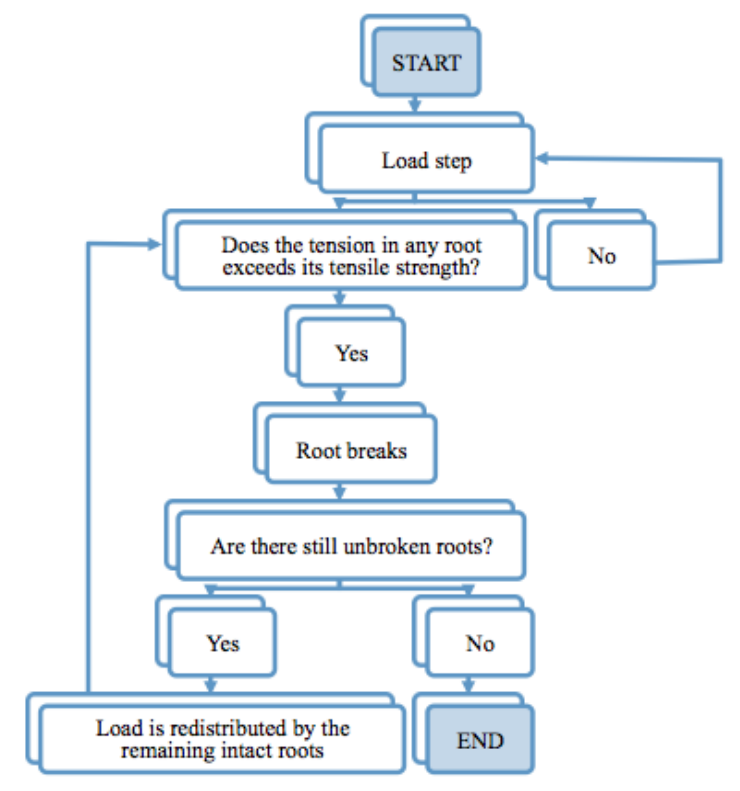

Fig. 3 Flowchart for the FBM computation

The load can be distributed by the roots according to three different criteria as a function of the cross section area of the roots, as a function of the root diameter or equally distributed by all the intact roots (Mao et al. 2012). Thomas and Pollen-Bankhead (2010) suggested that the load distribution by roots in FBM models should be made equally by all the roots (third criteria) because it showed to be more conservative in the estimation of soil shear strength reinforcement. In fact, different load partition methods varied the peak root-reinforcement value by up to $60 \%$.

When a root breaks, the load is redistributed by the remaining unbroken roots. It can follow two approaches: Global load sharing (GLS), in which the load is evenly distributed by the remaining roots; or Local load sharing (LLS), in which the load is redistributed by the neighbouring roots. The first is preferable because it requires less data, even though the real behaviour is somewhere between those two (Thomas and Pollen-Bankhead 2010).

Equation (4) is used to calculate additional root cohesion assuming that the load (force) is equally distributed by all the roots. First, the roots are ordered from the strongest to the weakest ( 1 to $N$ ), so that the breakage sequence upon loading is known. The index $j$ represents the weakest root that is still intact upon loading of the root bundle of $\mathrm{N}$ roots. Therefore, $R A R_{j}$ refers to the RAR of the root $j$ and $t_{r, j}$ is the strength of the weakest intact root. Therefore, the bundle resistance is equal to the resistance of weakest root multiplied by the number of intact roots (also represented by $j$ ). The value obtained from (4) is substituted into (2) to obtain the root reinforcement.

$$
t_{r}=\max \left(t_{r, j} \cdot R A R_{j} \cdot j\right)
$$

\section{Root Bundle Model}

The Root Bundle Model (RBM), proposed by Schwarz et al. (2010b), is an FBM extension in which a strain step loading approach is used. In this way a more realistic implementation of root mechanical effects in numerical models for slope stability calculations is provided (Vergani et al. 2014). The displacements are imposed equally in all $\mathrm{N}$ roots composing the bundle (Schwarz et al. 2010b). For 
each displacement increment, the load taken by the bundle is the sum of the stresses in all the roots, as long as the tension in each individual root does not exceed the maximum tensile strength. Root pull out is also admitted in the first formulation of this model.

The total force in the bundle $\left(F_{t o t}\right)$ is a function of displacement $\Delta x$ given by (5), where $F_{i}(\Delta x)$ is the maximum pull out force allowed in the root belonging to the diameter class $i, N$ is the number of diameter classes and $n_{i}$ is the number of roots of diameter class $i$.

$$
F_{\text {tot }}(\Delta x)=\sum_{i=1}^{N} F_{i}(\Delta x) \cdot n_{i}
$$

Schwarz et al. (2013) proposed changes in the previous RBM in order to take into account the strength variability within a root diameter class using a statistical approach by introducing a Weilbull survival function. This function is a complementary cumulative distribution function that captures the failure probability of a complex system beyond a threshold. This other model is referred as RBMw.

Schwarz et al. (2013) assumed that the probability of a root to survive $S\left(\Delta x^{*}\right)$ is given by (6):

$$
S\left(\Delta x^{*}\right)=\exp \left[-\left(\frac{\Delta x^{*}}{\lambda^{*}}\right)^{\omega}\right]
$$

where $\omega$ is the Weibull exponent, $\lambda^{*}$ is the scaling factor and $\Delta x^{*}$ is the normalized displacement. The exponent $\omega$ represents the relative variability of root strength in relation to an optimized root diameter (Schwarz et al. 2013; Vergani et al. 2014). High $\omega$ values (e.g. $\omega=100$ ) represent low variability of root mechanical behaviour resulting into displacement-force relations with sharp peaks correspondent to the failure of each root diameter class, as in Fig. 4. Decreasing values of $\omega$ traduce increasing variability of root strength within a diameter class and consequently a lower maximum force. Details on the calibration of the survival function can be found in Schwarz et al. (2013).

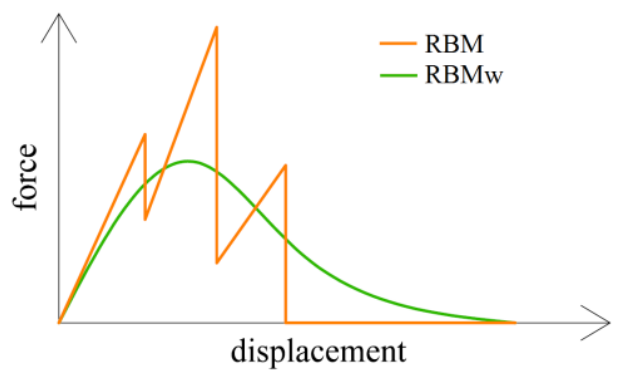

Fig. 4 Scheme of the RBM and RBMw outputs of a root bundle of three diameter classes

The tensile force of a bundle of roots $F_{\text {tot }}(\Delta x)$ at each incremental displacement $\Delta x$ is obtained by summing the force contribution of each root class $F\left(\phi_{\Phi}, \Delta x\right)$, given by (7), multiplied by the number of roots of that class $n_{\Phi}$ and by the survival function $S\left(\Delta x_{\Phi}^{*}\right)$, as in (8). Considering the force in a bundle as a sum of forces on individual roots accepts the assumption that the roots in the bundle do not interact with each other, which is considered an acceptable approximation by Giadrossich et al. (2013).

$$
\begin{gathered}
F\left(\phi_{\Phi}, \Delta x\right)=\frac{r \pi E_{0}}{4 L_{0}} \phi_{\Phi}{ }^{2+\beta-\gamma} \Delta x \\
F_{t o t}(\Delta x)=\sum_{\Phi=1}^{\Phi_{\max }} n_{\Phi} F\left(\phi_{\Phi}, \Delta x\right) S\left(\Delta x_{\Phi}^{*}\right)
\end{gathered}
$$

In these equations, $\beta$ and $E_{0}$ are calibration parameters associated to the Young's modulus of the root material relation with diameter, $\gamma$ and $L_{0}$ are calibration parameters associated to the root length 
relation with diameter, $r$ is the reduction coefficient to take into account the root tortuosity, $\Phi$ is the mean root diameter of each root class, $\Phi_{\max }$ is the maximum considered diameter and $\Delta x_{\Phi}^{*}$ is the normalized displacement. Apparently, RBM provides an additional force. But this force can be considered by unit area, providing this way an additional cohesion value. Indeed, switching from load increments to displacement increments provides important information for the calculation of root reinforcement. The peak root reinforcement does not generally occur at the same displacement as the soil peak strength, but for larger strain values (Nakamura et al. 2007; Schwarz et al. 2015). As a consequence, an increase of the displacement of the peak resistance and an increase of the residual resistance is observed as in Fig. 5 (Gray and Ohashi 1983; Abe and Ziemer 1991). Also, it opened a way for the development of constitutive laws to describe the root-permeated soil.

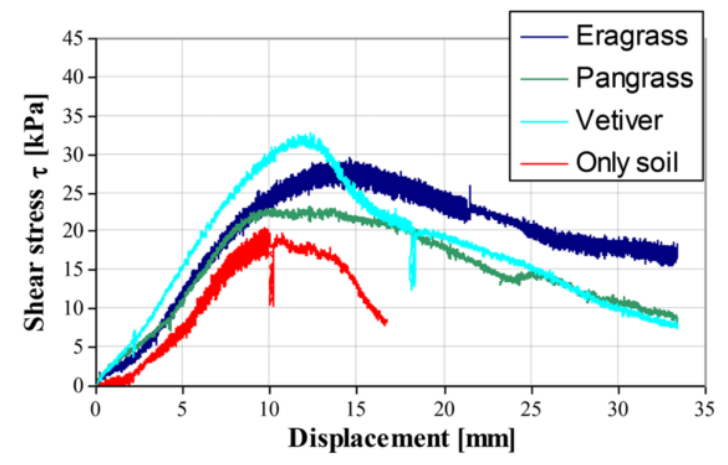

Fig. 5 Shear stress-displacement curves for soil samples with and without roots (Cazzuffi et al. 2014)

An analysis performed by Vergani et al. (2014) showed that the characterization of mechanical properties and root distribution is fundamental for the quantification of root reinforcement. Great variability in the estimated values were obtained by this author, that highlighted that the Weibull exponent applied to a general force-displacement curve leads to the estimation of conservative peak reinforcement values. Therefore, Vergani et al. (2014) stated that the use of the RBMw is an important improvement in the estimation of root reinforcement.

\section{Discussion on the Root "Breaking" Models}

Roots are loaded in different manners along the failure surface of a shallow landslide. At the crest, roots are subjected to pull out forces as a result of the tension cracks that may appear. Along the base of the unstable layer, shear actions are applied to the roots leading to pull out, breakage or buckling, depending on the orientation of the root. Finally, at the toe of the slope roots are under compression, failing by buckling (Schwarz et al. 2015). Each type of root failure is described in more detail in Dias et al. (2017), whose distinction is necessary in order to consider the resistance that the roots actually provide.

Not all the BMs were initially proposed to take into account different root failure mechanisms and for sure that the model by Wu (1976) in Fig. 2 does not represent all the presented situations. However, a clear and positive evolution of the root reinforcement calculation methods was observed in this regard. In this discussion section, it is highlighted the flexibility of the BMs and their potential.

As previously mentioned, the BMs provide the maximum reinforcement of a root bundle based on the resistance of each individual. This means that any critical load (breakage, slippage or buckling) can be used to study the root additional cohesion. Therefore, to have the tensile reinforcement mobilized in tension cracks, the pull out resistance of the roots is considered as input of the BMs, considering or not the slip out of the root (Schwarz et al. 2010a). To calculate the shear reinforcement, the previous results are applied to (2), in which the possibility of the root being under tension or compression is 
already considered in the parameter $k^{\prime}$. Finally, to obtain the reinforcement at the toe of the slope, the BM's input is the critical buckling load (Schwarz et al. 2015).

\section{Numerical Models}

Numerical methods have been used to estimate root reinforcement in the composite material. Yet, the description of root-soil interface and root large displacements are some of the limitations reported in the literature. The disadvantages of these methods are mainly related with the occurrence of fractures, as well as root breakage and slip out (Tiwari et al. 2013).

In the work of Mao et al. (2014), three-dimensional direct shear tests were simulated using both FEM and DEM models. Three different structural elements were used to simulate the roots and their interaction with the surrounding soil. The beam type roots presented tensile, compressive and bending resistance, the truss type presented tensile and compressive resistance and the cable type only mobilized tensile resistance. The calibration of these models is based on the results of direct shear tests on root-permeated soil.

Liang et al. (2015) produced an analytical model of a root and its soil interaction using existing $p-y$ curves for piles in a problem beam on elastic foundation under lateral loading. The study was executed for a shear zone at different depths by imposing displacements to the springs above the shear zone to simulate soil deformation, which is simpler from a computational point of view than using a continuum media. The ultimate resistance of the adopted $p$-y curve depends on the embedded depth of the root. Liang et al. (2015) found large relative soil-root deformations even for relatively small global slip of the rooted soil. In fact, at shallow depths, the soil that is pressed against the root move upward forming a wedge due to the low confining pressures. When the confining pressure increases, soil tends to contour the root. This way, the ultimate resistance of the soil surrounding the root is reached before the critical state of the soil. The beam model was validated by comparing the increase shear strength provided by roots based on direct shear tests and on the analytical model. The additional shear force was obtained from the numerical model of the beam by integrating the reaction force along the root.

Other FEM models of roots have been developed recently, such as Dupuy et al. (2007) and Fourcaud et al. (2008), however to study root anchorage when the plant is subjected to overturning actions (e.g. wind). Dupuy et al. (2007) produced a FEM model with the real root geometry represented by 2-node linear beam elements. A great computational effort was necessary to produce such analysis and it is unthinkable to extend such analyses for an entire slope. The interaction between the structural element and the soil is considered rigid based on field observations (Dupuy et al. 2007). Therefore, it is assumed that root slippage does not directly occur at the root-soil interface but in the nearest neighbouring soil around the roots (Mickovski et al. 2011; Mao et al. 2014).

\section{Application of Root Reinforcement to Slope Stability}

The calculation of the root reinforcement previously presented is then applied to access the safety factor (SF) associated to a slope. Two main approaches are the following ones: limit equilibrium (LE) and finite element method (FEM). In the following examples of application of those methods are presented.

\section{Limit Equilibrium}

Greenwood (2006) presented a software for the calculation of SF using LE analysis by the method of slices. The software allows a comparison of different computation methods, such as Bishop, Janbu, Fellenius, Simple and Greenwood. The inclusion of the vegetation effect is made by means of a force 
applied to the base of the slices and it is recommended by the author the lowering of the groundwater table by $10 \mathrm{~cm}$.

Even though Greenwood (2006) has suggested this software for preliminary analysis, some researchers, such as Danjon et al. (2008), Genet et al. (2010) and Sonnenberg et al. (2010), have been using it to investigate the influence of vegetation on slope stability for being user-friendly. Nevertheless, Stokes et al. (2009) highlighted that this method tends to overestimate the SF.

Other commercial software based on the slices method, such as GeoSlope, have been used to calculate the safety factor, as in the work of Tardio and Mickovsli (2015), where a surface soil layer is introduced in the model with the mechanical properties of a rooted soil.

Bischetti et al. (2010) investigated the effect of brush layering on slope stability using an infinite slope model where a resistant force is provided by each brush live cutting inserted in the soil. In the work of Nakamura et al. (2007), where roots were modelled according to Nghiem et al. (2003), the reinforcement provided by the roots is introduced in the infinite slope model with planar failure surface and the $S F$ is given by (9), where $w$ represents the soil weight of the slice per unit area, $u$ is the pore pressure at the base of the slice per unit area, $c$ is the soil cohesion, $\Delta S_{R c}$ is the increment of cohesion resultant from the presence of roots and $\alpha$ is the inclination of the slope. This is the simplest model.

$$
S F=\frac{(w \cos \alpha-u) \tan \phi+\left(c+\Delta S_{R c}\right)}{u \sin \alpha}
$$

However, it is known that roots reinforcement is only mobilized for greater displacements than the yielding strain of the soil (Gray and Ohashi 1983). Considering LE analysis seems, for this reason an approach which is not appropriate.

\section{Finite Element Method}

Root reinforcement is always represented as an increase of cohesion in the surface soil layer (Tiwari et al. 2013) or in the regions where vegetation is present (Genet et al. 2008; Mao et al. 2014; Liang et al. 2015). However, the method of calculation of such cohesion differs. In the case of Liang et al. (2015), the root reinforcement is obtained from a numerical model. On the other hand, Genet et al. (2008) used "breaking" models.

Genet et al. (2008) produced a FEM model using a commercial software to investigate how the root cohesion could influence the SF of the slope in unsaturated conditions. The root cohesion was calculated as a function of the depth and distance from the stem using W\&W. Similarly, Liang et al. (2015) used blocks of root-permeated soil material with increasing cohesion in depth in a 2D FEM model of the slope to investigate the root influence on the behaviour of a slope. In this case, the computation of the roots additional cohesion required the analyses of $p$-y curves for different depths of the failure surface, root diameters and group effect of a bundle of roots by comparing direct shear tests performed on spread roots.

Mao et al. (2014) produced a slope stability analysis using a 3D FEM software. The displacements and stresses at each node as well as the SF were calculated to access the influence of different roots densities and distributions on slope stability. The influence of the roots was considered as an additional root cohesion calculated using different root "breaking" models. However, the innovation in this work was that the properties of the soil were assigned individually to each node of the mesh using an algorithm allowing the root cohesion varying vertically and horizontally. 
Even though developments have been made on the estimation of the root cohesion, researchers insist on using W\&W, which is known to overestimate root reinforcement. Moreover, FEM enables the gradual mobilization of the root reinforcement with increasing displacement, but in the examples here presented root influence takes the form of an additional cohesion, which means that the full potential of FEM has not yet been used.

\section{Conclusions}

Roots are loaded in different manners along a failure surface of a shallow landslide. At the crest, tension cracks may appear and roots are subjected to pull out forces. Along the base of the unstable layer, shear actions are applied to the roots. At the foot of the slope, roots are under compression, failing by buckling (Schwarz et al. 2015). Due to mobilization of roots tensile strength, an increase of cohesion occurs into the soil, even though as influence on the frictional term for low confining pressures has been observed.

Most of the proposed methods to evaluate root reinforcement only assume root breakage, which leads to overestimation of the root reinforcement. Nevertheless, these methods are flexible enough to consider other types of root failure depending on what the ultimate resistance is considered to be. The evolution of these root "breaking" models lead to formulations that include the root resistance variability, as well as the possibility of investigating the displacement at which roots fail and its correspondent root reinforcement value.

Numerical models also have been used to study the effects of roots on the resistance of rootpermeated soil by using structural elements to represent the fibres. The advantage of such approach is in considering soil-root interaction, whose representation can still be improved.

Finally, a slope stability analysis can be produced considering the root reinforcement. The representation of this reinforcement can be through macro models or root-soil interaction models. The representation of a root system with structural elements is rather complex and computationally demanding, so the representation of the roots is made using an equivalent material with the same properties of root-permeated soil. LE and FEM models were used. In LE models, that tend to overestimate the SF, the root reinforcement is considered as an additional cohesion or resistant force at the base of the sliding layer is used. In the case of FEM, blocks or layers of soil are represented where the effect of the presence of roots is incorporated by an additional cohesion, not taking advantage of the full potential of the method.

\section{Acknowledgements}

The authors wish to acknowledge the support of the European Commission via the Marie SkłodowskaCurie Innovative Training Networks (ITN-ETN) project TERRE 'Training Engineers and Researchers to Rethink geotechnical Engineering for a low carbon future' (H2020-MSCA-ITN-2015-675762).

\section{References}

Abe K, Ziemer RR (1991) Effect of tree roots on a shear zone: modelling reinforced shear stress. Can J For Res 21(7):1012-1019

Belfiore G, Urciuoli G (2004) Analisi Del Contributo Meccanico Delle Radici Alla Resistenza Del Terreno. Incontro Annuale dei Ricercatori di Geotecnica 2004, 7-9 luglio 2004. Chieti, Italy (in Italian) 
Bischetti GB, Chiaradia EA, D'Agostino V, Simonato T (2010) Quantifying the effect of brush layering on slope stability. Ecol Eng 36(3):258-264

Cazzuffi D, Cardile G, Gioffrè D (2014) Geosynthetic engineering and vegetation growth in soil reinforcement applications. Transp Infrastruct Geotechnol 1:262-300

Danjon F, Barker DH, Drexhage M, Stokes A (2008) Using three-dimensional plant root architecture in models of shallow-slope stability. Ann Bot 101:1281-1293

Dias AS, Pirone M, Urciuoli G (2017) A review on the types of root failures along the failure surface of a shallow landslide. In: Proceedings of 4th WLF, 29 May-2 June 2017. Ljubljiana, Slovenia

Dupuy LX, Fourcaud T, Lac P, Stokes A (2007) A generic 3D finite element model of tree anchorage integrating soil mechanics and real root system architecture. Am J Bot 97(9):1506-1514

Fourcaud T, Ji JN, Zhang ZQ, Stokes A (2008) Understanding the impact of root morphology on overturning mechanisms: a modelling approach. Ann Bot 101(8):1267-1280

Genet M, Kokutse N, Stokes A, Fourcaud T, Cai X, Ji J, Mickovski S (2008) Root reinforcement in plantations of Cryptomeria japonica D. Don: effect of tree age and stand structure on slope stability. For Ecol Manage 256:1517-1526

Genet M, Stokes A, Fourcaud T, Norris JE (2010)The influence of plant diversity on slope stability in a moist evergreen deciduous forest. Ecoleng 36(3): 265-275

Giadrossich F, Schwarz M, Cohen D, Preti F, Or D (2013) Mechanical interactions between neighbouring roots during pullout tests. Plant Soil 367:391-406

Graf F, Frei M, Böll A (2009) Effects of vegetation on the angle of internal friction of a moraine. Forest Snow Landscape Res 82 (1):61-77

Gray DH, Ohashi H (1983) Mechanics of fiber reinforcement in sand. J Geotech Eng 109(3):335-353

Greenwood JR (2006) SLIP4EX-a program for routine slope stability analysis to include the effects of vegetation, reinforcement and hydrological changes. Geotech Geol Eng 24:449-465

Liang T, Knappett JA, Duckett N (2015) Modelling the seismic performance of rooted slopes from individual root-soil interaction to global slope behaviour. Géotechnique 65(12):995-1009

Mao Z, Saint-André L, Genet M, Mine F-X, Jourdan C, Rey H, Courbaud B, Stokes A (2012) Engineering ecological protection against landslides in diverse mountain forests: choosing cohesion models. Ecol Eng 45:55-69

Mao Z, Yang M, Bourrier F, Fourcaud T (2014) Evaluation of root reinforcement models using numerical modelling approaches. Plant Soil 381:249-270

Mickovskia SB, Stokes A, van Beek R, Ghestem M, Fourcaud T (2011) Simulation of direct shear tests on rooted and non-rooted soil using finite element analysis. Ecol Eng 37:1523-1532

Nakamura H, Nghiem QM, Iwasa N (2007) Reinforcement of tree roots in slope stability: a case study from the Ozawa slope in Iwate Prefecture, Japan. Eco- Ground Bio-Eng Use Veg Improve Slope Stab 103:81-90

Nghiem MQ, Nakamura H, Shiraki K (2003) Analysis of root reinforcement at slip surface. J Jpn Landslide Soc 40(4):44-52 
Pollen N, Simon A (2005) Estimating the mechanical effects of riparian vegetation on stream bank stability using a fiber bundle model. Water Resour Res 41(7):1-11

Schwarz M, Giadrossich F, Cohen D (2013) Modelling root reinforcement using a root-failure Weibull survival function. Hydrol Earth Syst Sci 17(11):4367-4377

Schwarz M, Cohen D, Or D (2010a) Root-soil mechanical interactions during pullout and failure of root bundles. J Geophys Res 115:1-19

Schwarz M, Lehmann P, Or D (2010b) Quantifying lateral rootreinforcement in steep slopes-from a bundle of roots to tree stands. Earth Surf Proc Land 35(3):354-367

Schwarz M, Rist A, Cohen D, Giadrossich F, Egorov P, Büttner D, Stolz M, Thormann J-J (2015) Root reinforcement of soils under compression. J Geophys Res Earth Surf 120:2103-2120

Sonnenberg R, Bransby MF, Hallett PD, Bengough AG, Mickovski SB, Davies MCR (2010) Centrifuge modelling of soil slopes reinforced with vegetation. Can Geotech J 47(12):1415-1430

Stokes A, Atger C, Bengough AG, Fourcaud T, Sidle RC (2009) Desirable plant root traits for protecting natural and engineered slopes against landslides. Plant Soil 324:1-30

Tardío G, Mickovski SB (2015) Method for synchronisation of soil and root behaviour for assessment of stability of vegetated slopes. Ecol Eng 82:222-230

Thomas RE, Pollen-Bankhead N (2010) Modelling root-reinforcement with a fiber-bundle model and Monte Carlo simulation. Ecol Eng 36:47-61

Tiwari RC, Bhandary NP, Yatabe R, Bhat DR (2013) New numerical scheme in the finite-element method for evaluating the root-reinforcement effect on soil slope stability. Géotechnique 63 (2):129-139

Vergani C, Schwarz M, Cohen D, aThormann J-J, Bischetti GB (2014) Effects of root tensile force and diameter distribution variability on root reinforcement in the Swiss and Italian Alps. Can J For Res 44 (11):1426-1440

Veylon G, Ghestem M, Stokes A, Bernard A (2015) Quantification of mechanical and hydric components of soil reinforcement by plant roots. Can Geotech J 52:1839-1849

Wu TH (1976) Investigation of landslides on Prince of Wales Island, Alaska. Columbus, $\mathrm{OH}$ : Department of Civil Engineering, Ohio State University; Geotechnical Engineering Rep. 5. 94 p

Wu TH (2013) Root reinforcement of soil: review of analytical models, test results, and applications to design. Can Geotech J 50:259-274

Wu TH, McOmber RM, Erb RT, Bear PE (1988) Study of soil-root interaction. J Geotech Eng 114(12):1351-1375

Zhang C, Chen L, Liu Y, Ji X, Liu X (2010) Triaxial compression test of soil-root composites to evaluate influence of roots on soil shear strength. Ecol Eng 36:19-26 